\title{
Optical Coherence Tomography for Bladder Cancer - Ready as a Surrogate for Optical Biopsy? - Results of a Prospective MONO-CENTRE STUDY
}

\author{
A. Karl ${ }^{1}$, H. Stepp 2 , E. Willmann ${ }^{1}$, A. Buchner ${ }^{1}$, Y. Hocaoglu ${ }^{1}$, C. Stief ${ }^{1}$, S. Tritschler ${ }^{1}$ \\ ${ }^{1}$ Urologische Klinik und Poliklinik der Ludwig-Maximilians-Universität München, Campus Großhadern, Munich, Germany, \\ ${ }^{2}$ LIFE Center, Campus Großhadern, Munich, Germany
}

\begin{abstract}
Introduction: New modalities like Optical Coherence Tomography (OCT) allow non-invasive examination of the internal structure of biological tissue in vivo. The potential benefits and limitations of this new technology for the detection and evaluation of bladder cancer were examined in this study.

Materials and Methods: Between January 2007 and January 2008, 52 patients who underwent transurethral bladder biopsy or TUR-BT for surveillance or due to initial suspicion of urothelial carcinoma of the bladder were enrolled in this study. In total, 166 lesions were suspicious for malignancy according to standard white light cystoscopy. All suspicious lesions were scanned and interpreted during perioperative cystoscopy using OCT. Cold cup biopsies and/or TUR-B was performed for all these lesions. For this study we used an OCT-device $\left(\right.$ Niris $^{\circledR}$, Imalux ${ }^{\circledR}$, Cleveland, US), that utilizes near-infrared light guided through a flexible fibre-based applicator, which is placed into the bladder via the working channel of the cystoscope. The technology provides high spatial resolution on the order of about $10-20 \mu \mathrm{m}$, and a visualization of tissue to a depth of about $2 \mathrm{~mm}$ across a lateral span of about 2 $\mathrm{mm}$ in width. The device used received market clearance from the FDA and CE approval in Germany. The diagnostic and surgical procedure was videotaped and analyzed afterwards for definitive matching of scanned and biopsied lesion. The primary aim of this study was to determine the level of correlation between OCT interpretation and final histological result.

Results: Of 166 scanned OCT images, 102 lesions $(61.4 \%)$ matched to the same site where the biopsy/TUR-BT was taken according to videoanalysis. Only these video-verified lesions were used for further analysis. Of all analyzed lesions 88 were benign (inflammation, edema, hyperplasia etc.) and 14 were malignant (CIS, Ta, T1, T2) as shown by final histopathology.

All 14 malignant lesions were detected correctly by OCT. Furthermore all invasive tumors were staged correctly by OCT regarding tumor growth beyond the lamina propria. There were no false negative lesions detected by OCT. Sensitivity of OCT for detecting the
\end{abstract}

presence of a malignant lesion was 100\% and sensitivity for detection of tumor growth beyond the lamina propria was $100 \%$ as well. Specificity of OCT for presence of malignancy was $65 \%$, due to the fact that a number of lesions were interpreted as false positive by OCT.

Conclusion: As a minimally invasive technique, OCT proved to have extremely high sensitivity for detection of malignant lesions as well as estimation of whether a tumor has invaded beyond the lamina propria. However, specificity of OCT within the bladder was impaired $(65 \%)$, possibly due to a learning curve and/or the relatively low spatial resolution and visualization depth of the OCT technology. Further studies and technical development are needed to establish an adequate surrogate for optical biopsy.

Key words: Bladder cancer, diagnosis, minimally invasive, optical biopsy, optical coherence tomography

\section{INTRODUCTION}

Early diagnosis and tumor specific follow up of patients with bladder cancer is essential for an optimal and timely induction of an individualized therapy. Standard non-invasive diagnostic techniques like ultrasound (US), computed tomography (CT) or magnetic resonance imaging (MRI) give only restricted information regarding the entity of a lesion (malignant or benign) within the bladder. Furthermore these techniques do not give reliable information regarding tumor invasion (superficial growth, invasion of the lamina propria, invasion of musculature etc.). In many cases invasive bladder biopsy, or TUR-BT, is needed to diagnose a specific disease and exclude or confirm malignant tumor presence. The additional use of Optical Coherence Tomography (OCT) could enable the examiner to decide whether a lesion must be biopsied or whether it can be confidently determined as benign. OCT as an optical surrogate for biopsy could eliminate the need for primary surgical intervention and anaesthesia for diagnosis and follow up of bladder lesions. This prospective mono-center study aims to evaluate the clinical use and feasibility of OCT in the detection of bladder cancer. 


\section{MATERial AND Methods}

Between January 2007 and January 2008, 52 patients who underwent transurethral bladder biopsy or TURBT for surveillance or due to initial suspicion of urothelial carcinoma of the bladder were enrolled in this study. Patients' age ranged from 21 to 91 years. This study included 32 patients with a positive history of urothelial cancer and 20 patients with initial suspicion for malignancy (positive cytology, gross hematuria, suspicious cystoscopical finding etc.). Excluded from this study were patients with an age below 18 years, pregnant patients and patients without informed consent.

All examinations followed a standardized protocol. First every patient underwent a cystoscopy in white light using a standard cystoscope sheath (25 Charrière) with an integrated working channel using $30^{\circ}$ degree optics, or sometimes $70^{\circ}$ degree optics (Storz ${ }^{\circledR}$, Tuttlingen, Germany) if needed. Before tissue removal (biopsy/TUR-BT), all suspicious lesions were scanned and evaluated during the procedure using OCT. In our investigation we used an OCT-device (Niris ${ }^{\circledR}$, Imalux ${ }^{\circledR}$, Cleveland, US) that utilizes near-infrared light guided through a flexible fibre-based applicator, which is placed into the bladder via the working channel of the cystoscope. OCT - scanning was performed using a $1300-\mathrm{nm} 10-\mathrm{mW}$ superluminescent diode with an applicator with an outer diameter of $2.7-\mathrm{mm}$. OCT scans with a duration of approximately 1.5 seconds each generated 200 x 200-pixel images. This enabled visualisation of surface tissue and sub-surface tissue microstructure. The technique provided high spatial resolution on the order of about $10-20 \mu \mathrm{m}$, and a visualization of tissue to a depth of about $2 \mathrm{~mm}$ across a lateral span of about $2 \mathrm{~mm}$ in width. OCT does not require a conducting medium and can therefore image through air or water $[7,8]$.

A specially designed documentation sheet was used to record the exact location of each lesion within the bladder and its morphology in white light (flat, papillary, solid etc.). The whole procedure of cystoscopy, OCT scanning and tissue removal was videotaped for later analysis. Use of the diagnostic device was approved by the ethics committee of the LMU Munich, Germany.

\section{RESULTS}

Of 166 scanned OCT images, 102 lesions (61.4\%) matched to the same site where the biopsy/TUR-BT was taken according to videoanalysis. Only these videoverified lesions were used for further statistical analysis. Of the evaluable lesions, 88 were benign (acute inflammation $\mathrm{n}=9$, chronic inflammation $\mathrm{n}=44$, edema $\mathrm{n}=$ 3 , normal tissue $\mathrm{n}=12$, scaring $=5$, hyperplasia $\mathrm{n}=1$, metaplasia $\mathrm{n}=6$, low grade dysplasia $\mathrm{n}=4$, intraepithelial benign findings $n=4$ ) and 14 were malignant (high grade dysplasia $\mathrm{n}=2$, CIS $\mathrm{n}=1$, Ta $\mathrm{n}=6$, T1 $\mathrm{n}$ $=3$, T $2 \mathrm{n}=2$ ) as shown by final histopathology.

All 14 histologically proven malignant lesions were detected correctly by OCT. Furthermore all invasive tumors were staged correctly by OCT as having tumor growing beyond the lamina propria. No lesions were

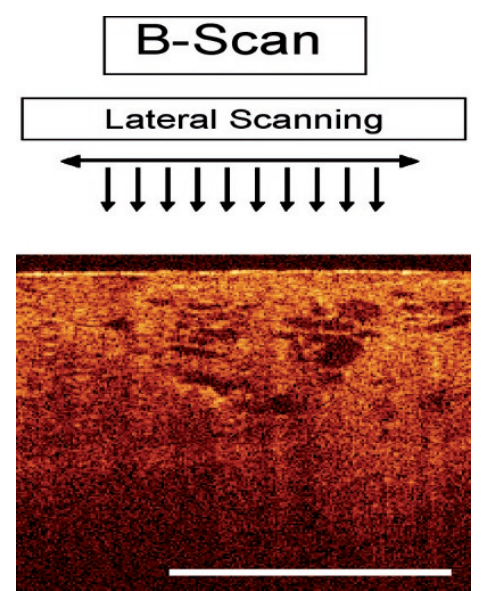

Image 1: OCT Scan: Lateral scanning provides two-dimensional images, that are called analogous to ultrasound B-Scan.

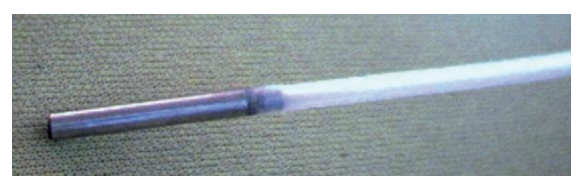

Image 2: OCT probe (Ø $2.7 \mathrm{~mm})$, inserted into the bladder via the working channel of the cystoscope sheath.

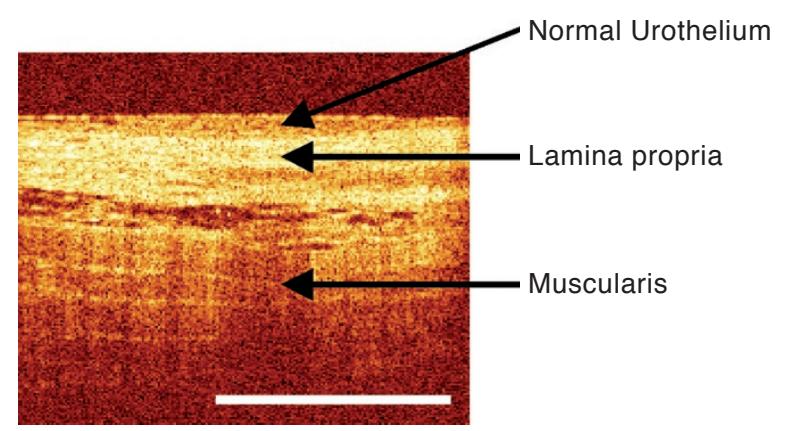

Image 3: Layers of a healthy urinary bladder as generated by OCT.

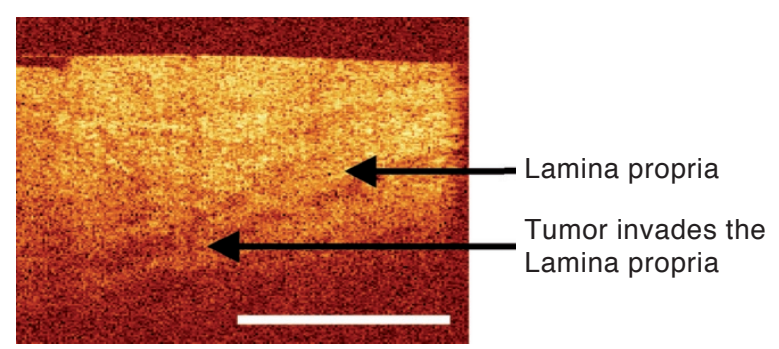

Image 4: OCT Scan: Tumor invades the Lamina propria.

false negative as detected by OCT. Of 88 histologically benign lesions, 31 turned out to be false positive and 57 to be correctly negative in OCT. Sensitivity of OCT regarding the presence of a malignant lesion was $100 \%$ and sensitivity regarding tumor growth beyond the lamina propria was $100 \%$ as well. Specificity of OCT for presence of malignancy was $65 \%$. False positive $(\mathrm{n}=31)$ lesions were misinterpreted edemas, 
Table 1. Sensitivity and Specificity of OCT regarding the presence of a malignant tumor.

\begin{tabular}{l|l|l}
\hline & \multicolumn{2}{|l}{ „Gold Standard“ } \\
\hline & $\begin{array}{l}\text { Histology } \\
\text { positive }\end{array}$ & $\begin{array}{l}\text { Histology } \\
\text { negative }\end{array}$ \\
\hline OCT positive & 14 & 31 \\
\hline OCT negative & 0 & 57 \\
\hline
\end{tabular}

chronic inflammations or scars. Regular layers of these lesions were not clearly demarked using OCT. Tumor invasion beyond the lamina propria was detected correctly in all five cases. In 16 benign lesions, an invasion of the lamina propria was suspected in OCT, but not proven in final histology.

\section{DISCUSSION}

Standard non-invasive imaging techniques like conventional ultrasonography, C'T, and MRI have significant limitations due to low spatial resolution and subsequent inability to provide sufficient histopathologic detail $[5,6]$. Determination of a lesion as clearly superficial is barely possible, as well as accurate judgement of the invasion depth, unless the tumor is far advanced or widespread. Furthermore the classification of a tumorous lesion within the bladder to be malignant or benign is nearly impossible using standard imaging techniques. The concordance of CT finding and pathological result of a cystectomy specimen is only between $65 \%$ to $80 \%$ [1].

Even more difficult is the exact evaluation of a non-muscle invasive tumor of the human bladder using these techniques. There is no way to use ultrasonography, CT, or MRI for grading of a tumor. Currently, a histopathologically examined actual specimen in needed for every final diagnosis Obtaining a specimen requires an invasive procedure that is often performed with regional or general anesthesia. This procedure is not only a physical and psychological stress for the patient, but is also a high financial burden for the health care system.

For a long time now, there has been a need for a minimally invasive technology that permits differentiation of malignant and benign findings in the bladder, and moreover to judge the invasion depth of a tumor. Such a technology should provide the diagnostic information as soon as possible, hopefully "online" during cystoscopy. The scanned image should be interpretable by the examining physician himself, so that the necessity of proceeding to an invasive procedure can be immediately assessed. The advantage of a technology like this is the ability of the urologist to make an immediate diagnosis in an outpatient setting, thus avoiding the need to take a biopsy and subject the patient to an invasive procedure under anesthesia. Such an approach would reduce not only risk for the patient, but could also save expenses for the health care system.

Optical coherence tomography is already successfully in use in the fields of Ophthalmology, Dermatology or Cardiology [2-4].
In Urology, the first studies using this technology are being published. In different animal trials [5, 6] as well as in humans [7-9] it was possible to detect Carcinoma in situ which is difficult to diagnose with white light cystoscopy. Papillary tumors could also be detected by OCT. Manyak et al. [10] analyzed 261 OCTScans in 24 patients. Of 87 OCT scanned areas, 29 of 36 visually suspicious lesions and 35 of 35 visually unsuspicious lesions were correctly judged using OCT. Of 16 areas with papillary urothelial tumors, all 16 were detected correctly as tumors, whereas 9 of 10 were diagnosed correctly to be invasive. In this study, OCT had a sensitivity of $100 \%$ and a specificity of $89 \%$. The positive predictive value was $75 \%$ and the negative predictive value was $100 \%$.

OCT is also currently used in the differentiation of the neurovascular bundle during radical prostatectomy in animal models [11] as well as in human trials [12].

Despite promising initial reports, the currently used, in vivo, OCT devices have certain limitations, which were apparent during this study. The $2 \mathrm{~mm}$ invasion depth of the technology is not always sufficient to judge the invasion depth of a tumor, especially if the diameter of a tumor is already larger than 2 $\mathrm{mm}$ before reaching the area of the lamina propria, making the judgement of muscle invasion impossible. Unfortunately there are also solid and papillary tumors, where invasion depth cannot be judged sufficiently.

Moreover the resolution of currently available in vivo devices does not allow the differentiation of a single cell layer or the morphology of a single cell. One new technology that proved to have a far higher resolution was confocal lasermicroscopy. In vitro studies showed the visualization of single cells which gives the opportunity to judge the grading of a cell [13], but the invasion depth of this technique is far below that of OCT. Using OCT, imaging of tissue structures has a resolution 20-times higher than that of high resolution-ultrasound. Thus, depending on the OCT device used (in vitro / in vivo), structures as small as $4-20 \mu \mathrm{m}$ can be visualized.

Accurate differentiation between inflammative tissue and carcinoma in situ is not sufficiently possible yet by OCT. Also the classification (edema, CIS, scar tissue etc.) of a lesion using OCT turns out to be extremely difficult.

The high rate of drop-outs in our study, lesions in which biopsy and scanning area could not be matched sufficiently using later video-analysis, can be explained by the need to change the cystosope sheath and biopsy sheath between each lesion within the bladder. To avoid unnecessary urethral damage only one change was allowed between the cystoscopy sheath, which had to be used for OCT due to a larger diameter of the working channel, and the resectoscope sheath, which was used for biopsy or TUR-BT. So if there was more than one lesion within the bladder scanned by OCT, it was extremely difficult to find the exact site (probe diameter of $2.7 \mathrm{~mm}$ ) again after changing the sheaths. Since no other marking tool was used on the scanned OCT sites, only slightly more than $60 \%$ of biopsyand OCT scanned sites were matching according to the later videoanalysis. 
To optimize conditions for further studies, a sheath which allows the performance of OCT and biopsy/TUR-BT at the same time is needed. Otherwise a marking tool should be used to avoid the high drop-out rate we experienced in this study. Despite these drawbacks, OCT proved to be a promising technology that may help to avoid invasive procedures for lesions which could be clearly defined as benign. Furthermore, with OCT it was possible to classify tumors as invasive or going beyond the lamina propria. With a higher resolution and a greater invasion depth, OCT technology could potentially be an excellent surrogate for optical biopsy. A study combining OCT and confocal lasermicroscopy would be interesting. It would be interesting to use OCT technology to judge especially flat lesions like carcinoma in situ, since the invasion depth would be sufficient for this type of lesion. Also, evaluation of the resection ground of a tumor could be checked for completeness of tumor removal and sufficient removal of musculature.

\section{CONCLUSION}

As a minimally invasive technology for the detection of bladder cancer, OCT showed an extremely high sensitivity for malignant lesions as well as estimation of whether a tumor has invaded beyond the lamina propria. However, specificity of OCT within the bladder was impaired $(65 \%)$, possibly due to a learning curve and/or the relatively low spatial resolution and visualization depth of the OCT technology used. Further studies and technical development are needed to establish an adequate surrogate for optical biopsy.

\section{REFERENCES}

1. Paik ML, Scolieri MJ, Brown SL, et al. Limitations of computerized tomography in staging invasive bladder cancer before radical cystectomy. J Urol. 2000;163(6): 1693-1696.

2. Costa RA, Skaf M, Melo LA Jr., et al. Retinal assessment using optical coherence tomography. Prog.Retin.Eye Res. 2006;25(3)325-353.

3. Gambichler T, Moussa G, Sand M, et al. Applications of optical coherence tomography in dermatology. J Dermatol Sci. 2005;40(2): 85-94.
4. Welzel J. Optical coherence tomography in dermatology: a review. Skin Res Technol. 2001;7(1): 1-9.

5. Pan Y, Lavelle JP, Bastacky SI, et al. Detection of tumorigenesis in rat bladders with optical coherence tomography. Med Phys. 2001;28(12): 2432-2440.

6. Wang ZG, Durand DB, Schoenberg M, et al. Fluorescence guided optical coherence tomography for the diagnosis of early bladder cancer in a rat model. J Urol. 2005;174(6: )2376-2381.

7. Karl A, Stepp H, Willmann E, et al. Optical coherence tomography (OCT): ready for the diagnosis of a nephrogenic adenoma of the urinary bladder? J Endourol. 2008; 22(11): 2429-2432.

8. Manyak MJ, Gladkova ND, Makari JH, et al. Evaluation of superficial bladder transitional-cell carcinoma by optical coherence tomography. J Endourol. 2005;19(5): 570574.

9. Zagaynova EV, Streltsova OS, Gladkova ND, et al. In vivo optical coherence tomography feasibility for bladder disease. J Urol. 2002;167(3): 1492-1496.

10. Manyak MJ, Gladkova ND, Makari JH, et al. Evaluation of superficial bladder transitional-cell carcinoma by optical coherence tomography. J Endourol. 2005;19(5): 570574.

11. Rais-Bahrami S, Levinson AW, Fried NM, et al. Optical Coherence Tomography of Cavernous Nerves: A Step Toward Real-Time Intraoperative Imaging During NerveSparing Radical Prostatectomy. Urology. 2008 Jul;72(1): 198-204.

12. Aron M, Kaouk JH, Hegarty NJ, et al. Second prize: preliminary experience with the Niris optical coherence tomography system during laparoscopic and robotic prostatectomy. J Endourol. 2007;21(8): 814-818.

13. Sonn GA, Mach KE, Jensen K, et al. Fibered confocal microscopy of bladder tumors: an ex vivo study. J Endourol. 2009;23(2): 197-201.

Received: October 20, 2009 / Accepted: November 3, 2009

Address for correspondence:

Alexander Karl, MD

Department of Urology

Ludwig-Maximilians-University Munich

Marchionistr. 15

81377 Munich

Germany

Tel.: $\quad+49$ 89/7095-0

Fax.: $\quad+4989 / 7095-8890$

E-mail: alexander.karl@med.uni-muenchen.de 